\title{
Reliability of MEMS for space applications
}

\author{
Herbert R. Shea* \\ Microsystems for Space Technologies Laboratory, Ecole Polytechnique Fédérale de Lausanne \\ (EPFL), rue Jaquet Droz 1, CH-2007 Neuchatel, Switzerland.
}

\begin{abstract}
With their extremely low mass and volume, low power consumption and tight integration with electronics, MEMS sensors and actuators are extremely appealing for reducing the size and mass of spacecraft without sacrificing functionality. In view of the harsh and remote environment of space, reliability and qualification is the crucial issues that are holding back MEMS from playing a larger role in space applications.

We examine how MEMS reliability is handled in commercial MEMS devices used in safety critical applications on earth and contrast the operating conditions on earth with those encountered during launch and in orbit. We explain the impact that vibration, mechanical and thermal shock, and radiation can have on MEMS devices fabricated using the most widespread silicon technologies. Accelerated tests adapted to space qualification are presented as a means to determine the major failure modes. Hermetic packaging is crucial to ensuring long-term reliability.
\end{abstract}

\section{INTRODUCTION}

MEMS have been commercially adopted in large volumes in a number of earth-bound applications, the most common being accelerometers for automotive (airbag) applications [1], pressure sensors for engine management [2], and micromirror arrays for display applications [3].

Combining low mass, low power consumption, small volume and possible integration with control and sense electronics, MEMS seem ideal for space applications. Considering the high reliability achieved on earth, the challenges of developing mechanisms for use in space, and the reduction in part number that highly integrated MEMS enable, it is conceivable that a spacecraft including carefully designed MEMS could be more reliable than the conventional solution.

Initially MEMS will serve simply as replacements for bulkier sensors (e.g., accelerometers or gyros). In the medium term MEMS technology will allow sub-systems (such as phased array antennae, attitude determination, optical switches) to be reduced significantly in size and mass. In the longer run MEMS can enable new classes of extremely small, intelligent, self managing and relatively low-cost batch-produced picosatellites consisting primarily of bonded MEMS devices.

In this paper we present a brief overview of the broad range of possible MEMS applications in space. We will then examine how MEMS reliability is handled in commercial MEMS devices used on earth and contrast the operating conditions on earth with those encountered during launch and in orbit. We explain the impact that vibration, mechanical and thermal shock, and radiation can have on MEMS devices fabricated using the most common silicon-based technologies. Accelerated tests adapted to space qualification are presented as a means of determining the major failure modes. We propose design rules for MEMS to avoid such failures, such as selective etching of dielectric for radiation hardness, material and suspension designs for high shock resistance (over $1000 \mathrm{G}$ ). Packaging is crucial to ensuring long-term reliability. Techniques for hermetic packaging of MEMS are discussed.

\section{OVERVIEW OF MEMS SPACE APPLICATIONS}

MEMS have been proposed for a number of space applications, either as lighter replacement parts or as entire new systems. A few MEMS components have been flown in space, mostly as technology demonstrators. We briefly list some of the more developed areas below, roughly in order of decreasing maturity. Certain topics such as power MEMS are not addressed in this paper.

*email: herbert.shea@epfl.ch ; phone :+41 327205584

Reliability, Packaging, Testing, and Characterization of MEMS/MOEMS V, edited by Danelle M. Tanner, Rajeshuni Ramesham, Proc. of SPIE Vol. 6111, 61110A, (2006) · 0277-786X/06/\$15 - doi: 10.1117/12.651008 
Inertial Navigation: accelerometers and gyroscopes are perhaps the most mature MEMS devices. Since these devices are available as COTS (commercial off the shelf) parts for commercial and military applications, it is likely that they can be re-qualified for space by de-rating (for de-rating procedure see ref [7]). There have been several studies [4,5,6] addressing this issue for space. COTS accelerometers have been shown to survive 1000 temperature cycles from $-65^{\circ} \mathrm{C}$ to $+150^{\circ} \mathrm{C}$, as well as 30,000 mechanical shocks of $2,000 \mathrm{G}$.

Bolometers: micromachining allows thermal isolation of small detectors, enabling both uncooled and cooled bolometer arrays to offer very high performance. Such devices are commercially available for earth-based IR detector applications. A MEMS bolometer from JPL/Caltech [8] is planned for the ESA/NASA Planck mission in 2007.

RF Switches and Variable Capacitors: MEMS technologies enables the fabrication of very compact low-loss RF switches, as well as capacitors with a large tuning range. Such devices are just starting to be commercially available, for example from Teravicta [9] (Austin, Texas, USA). RF switches were flown in space on the OPAL Picosats in 2000. They were stored in orbit for a year, and then successfully operated [10]. IMEC has led several studies into the reliability of RF MEMS for Space [11].

Optical Switching and Communication: The boom in optical MEMS between 1999 and 2002 led to the development of large optical switch matrices based on MEMS devices. There are ongoing ESA project on the development and qualification methodology of such MEMS switches for space. There have been studies of COTS parts [12], but no devices have been flown.

Propulsion: There are three main approaches to using MEMS for propulsion in space. One approach is to miniaturize an ion-thruster by micromachining and integrating the electron source, gas handling, nozzle and other components, see for example reference [13]. Another approach is to microfabricate arrays of microthrusters [14] which consist of micromachined cavities filled with solid propellant. On top of each cavity a micromachined hotplate is bonded to allow ignition of the microthrusters one at a time. Thirdly, one can use silicon machining to fabricate compact cold gas thrusters consisting of bonded Si chips to form a reaction chamber and compact nozzle. [15]

Bio and Microfluidics: SU8 and other polymers, as well as Pyrex and silica are used to fabricate micro-fluidic devices, including channels, nozzles and pumps. Such devices are principally being developed for medical or pharmaceutical (lab-on-a-chip) applications [18], but could eventually be used as part of a propulsion system.

\section{RELIABILITY OF MEMS AND FAILURE MODES}

\subsection{Overview}

To determine the reliability of MEMS, one must understand the root cause of all relevant failure modes using a rigorous physics-based approach. This starts with a test plan to reveal failure modes by applying drive conditions (e.g., overvoltage) or environmental conditions (e.g., humidity, thermal and mechanical shock) that could accelerate failures. Once one understands the failure modes, one can perform experiments to determine the acceleration factors, and run tests that can provide accurate estimates of the lifetime in a time much shorter than that lifetime (which could be of order hundreds of years for a well designed and packaged device). One may need to work with test structures designed to exhibit only one main failure mode if the MEMS device is too complicate to realistically accelerate certain failure modes. An important strategy is concurrent design, also referred to as design-for-reliability, as explained in reference [17]. The process is very iterative: once a failure mode is uncovered, the design, fabrication process, packaging or materials can then be improved to eliminate or minimize that failure mechanism.

Failure modes of MEMS devices can be categorized into a) mechanical and b) electrical failure modes. Much effort has been invested over the past 10 years into understanding mechanical failure modes, based on the intuitive assumption that it must be the moving parts that lead to failures and that electrical failure modes (and how to accelerate and avoid them) are well understood from the microelectronics industry.

It is the author's opinion (see reference [18]) that the mechanical design of MEMS has reached sufficient maturity that electrical failure modes often dominate, especially for high voltage designs, in large part because the thin films have been optimized for mechanical properties (e.g., ultra low stress) at the expensed of electrical ones (e.g., dielectric breakdown voltage). 
For some high volume and safety-critical applications (most notably accelerometers for airbag deployment in cars) reliability has been extensively studied and has yielded parts with failure rates at the ppm level of lifetimes of over 10 years [19]. For smaller volume markets where reliability is still crucial (telecom), extremely reliable MEMS have also been demonstrated. For example Lucent Technology has developed Si-based micromirrors with FIT rates of less than 50 (i.e., fewer than 50 failures in $10^{9}$ device hours).

\subsection{Most Common Failure Modes}

We summarize below some possible failure modes for MEMS devices, as well as a few possible design fixes. A comprehensive review can be found in [17] and [20] and references therein. Failure modes depend on the materials used for the device, the fabrication approach, the packaging, and of course the design. Most of the failure modes listed below can be eliminated through proper design and packaging. One should note however that designing for reliability can involve trade-offs, for instance sacrificing a degree of performance for longer lifetime, or using a more fabrication complex process to slow down device degradation.

Mechanical failure modes include:

- $\quad$ Stiction and wear

- Fatigue

- Plastic deformation

- Delamination

- Curvature change

- $\quad$ Shock and vibration induced fracture

Electrical failure modes include:

- Short and open circuits

- Arcing across small gaps

- ESD

- Dielectric charging

- Corrosion

It is beyond the scope of this paper to discuss in detail the techniques for mitigating the failure modes listed above. We note however that extensive design rules have been developed to make highly reliable silicon and metal MEMS structures. In particular it is now well known thanks to careful research $[21,22]$ how to make Silicon suspension beams that exhibit no fatigue and no plastic deformation. One must first ensure there is no metal on the silicon beam. To avoid fatigue (growth of cracks due to cyclic stress) once must operate in a dry ambient with maximum strains of less than $20 \%$ of yield strength. For example, using such principles, Lucent Technologies built and commercialized micromirrors that operated with no change in parameters (no fatigue and no creep) for over $2 \times 10^{10}$ cycles [23]. Figure 1 provides a summary of good design practices for developing a reliable silicon-based electrostatically-actuated MEMS micromirror (from reference [20]).

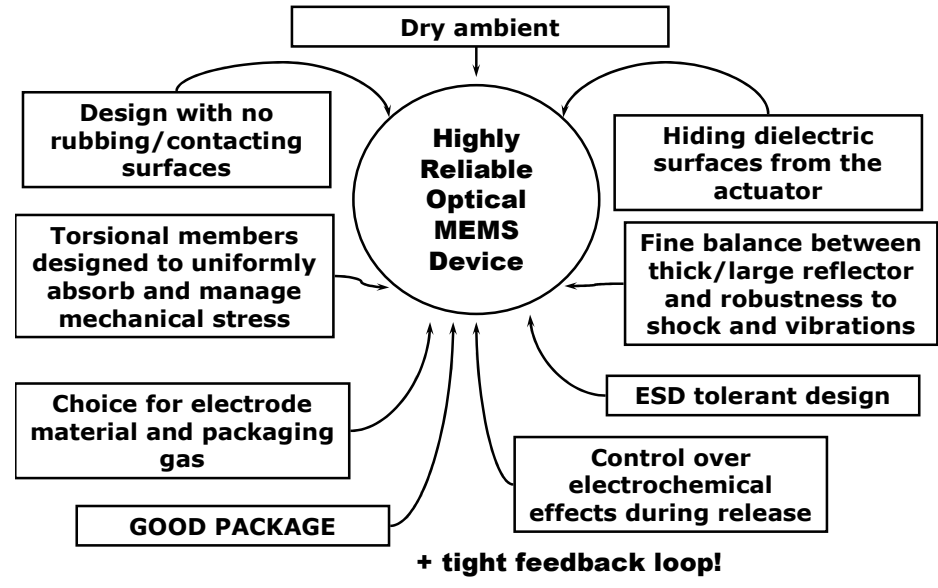

Figure 1. Schematic diagram of good design practices for electrostatically operated MOEMS devices. Most of the rules (in particular packaging and concurrent design) are applicable to all MEMS technologies. 


\subsection{Accelerated Tests}

The failure modes for microelectronic devices are well known (such as electromigration, solder fatigue), as are the accelerating factors (for instance current density and temperature for the case of electromigration, or thermal cycling for solder fatigue). This knowledge allows the long-term reliability of such devices to be rapidly evaluated by operating them under conditions that are more sever than normal so as to accelerate failures.

How can one perform accelerated testing on MEMS to develop the data needed to guarantee the device's long term reliability? This is a field that is still being explored for MEMS. It bears noting that humidity is a major accelerating for most mechanical and electrical failure modes. This means packaging (and possibly getters) is an essential component of device reliability. Indeed, despite being high volume products, TI's DMD chips are hermetically packaged, as are all devices for the telecom market. Shelf-life (storage) remains a challenging aspect to accelerate, and is also strongly tied to the quality of the packaging.

Determining qualification tests for MEMS requires a good understanding of the failure modes. If the modes are not identified and understood, the device might pass what appear to be stringent qualification tests, only to fail later. The challenge is that while certain aspect are now well understood (e.g., fatigue), others are still hard to model (such as stiction). Qualification tests are further complicated by the very diverse nature of MEMS devices: it is not appropriate to use the same qualification method on a polymer fluidic circuit as on a SiC MEMS accelerometer.

Accelerating factors (AF) for mechanical failures are listed below:

- Fatigue. AF: 1. number of cycles, 2. maximum applied strain, 3. humidity. Accurate models exist to extrapolate to the number of cycles to failure under nominal operating condition based on number of cycles to failure under accelerated conditions $[21,22,24,25]$

- Plastic Deformation (Creep). AF: 1. temperature, 2. applied strain. This is a field that is well understood. The homologous temperature provides a good guide to the magnitude of the expected creep for different materials.

- Stiction: AF: 1. humidity. This is a much more difficult effect to model, especially for shelf life. There are many mitigation techniques, including self-assembled monolayers (anti-stiction coatings) but few well established accelerated test techniques.

- Shock and Vibration induced fracture. 1. repeated mechanical shocks, 2. vibration on three axes. It may be necessary to use SEM or TEM to study the microstructure, as well as very accurate measurements of resonant frequency to look for the growth of minute cracks that could lead to later failures.

- Delamination: AF: 1. thermal shocks, 2. mechanical shocks.

We list below accelerating factors (AF) for electrical failures. These failure modes are similar to those in the microelectronics world. One must use caution however because MEMS devices often use combinations of materials not used in microelectronics, as well as high $(>100 \mathrm{~V})$ for many actuators.

- Short and open circuits: AF: 1. electric field, 2. temperature, 3. humidity

- Arcing across small gaps: AF: 1. electric field, 2. gas pressure and composition

- Dielectric Charging. AF: 1. electric field, 2. temperature, 3. radiation, 4. humidity

- Corrosion: AF: 1. humidity, 2. voltage and polarity (if anodic corrosion), 3. temperature

An intriguing question for MEMS devices is Burn-In. This is a standard process for microelectronics devices, designed to weed out devices with built-in weaknesses or defects, by operating the devices for a short period of time under harsh environmental or drive conditions. How is this best done for MEMS devices? Answering this question fully requires understanding the failures rate vs. time. This extensive data is only available for a few high volume parts (this information is rarely published). The TI DMD mirrors undergo an effective burn-in when tested after manufacturing.

\section{SPACE SPECIFIC RELIABILTY CONCERNS}

The ultra-high reliability MEMS parts (e.g. accelerometers for safety critical applications) used on earth are manufactured by the millions, which allows a careful statistical study to be performed. When coupled with root cause analysis of the failed parts, this allows a very complete picture of device reliability to be assembled. A major challenge for space applications is the possibly very limited total number of devices fabricated if a customized solution is used, as 
opposed to a COTS part. A limited supply of parts, or parts that are not particularly mature, complicates the qualification process.

We consider below radiation, vacuum, thermal shock and vibration as space-specific operating conditions, and their possible impact on MEMS reliability. Other possible considerations included atomic oxygen and plasmas.

\subsection{Radiation}

Even at the high end of space mission doses, the mechanical properties of silicon and metals are mostly unchanged (Young's modulus, yield strength not significantly affected). Silicon as a structural material is intrinsically radiation hard. This makes most MEMS devices radiation hard by default. Of much greater concern are the drive/control electronics, which may need to be shielded or built with a rad-hard design and components.

Radiation damage typically causes latch-up or single event upsets for electronics, or a continuous deterioration of optical coatings and lenses. Microelectronic circuits typically consist of millions of transistors, separated by small fractions of a micron, with very thin $(\mathrm{nm})$ dielectrics or gate oxides, and with each device's conductivity controlled by locally applying a gate voltage to doped semiconductors.

MEMS devices can operate on several principles, the most common being electrostatic, thermal, and piezo-electric. All those actuation schemes require a good electrical contact between a bond pad and the actuator (electrode) of the device, but the exact level of doping is not important as long as the material is sufficiently conductive. MEMS themselves typically have no p-n junctions or semiconducting regions where doping and carrier concentration plays an important role (except for some strain gauges) There are no active areas like a transistor, only zones where the electric potential needs to be well defined. The dielectric films are thick (of order a micron). This makes MEMS devices mostly insensitive to ionization or atomic displacement from radiation.

For MEMS devices the main failure mode at high radiation doses is the accumulation of charge in dielectric layers, which first causes a change in the calibration of the device (essentially by applying a quasi-constant electrostatic force), and ultimately can lead to complete failure by a short circuit or continuous actuation even at 0 volts. We discuss below the radiation levels needed to begin degrading performance, not the higher levels needed for complete device failure.

Few radiation tests have been performed on MEMS devices. It is clear that the design and materials play an important role in total acceptable dose. For example, micro-engines from Sandia National Labs in Albuquerque, NM, USA were reported to only change their behavior at doses of order 10 MRads [26]. Those devices did not contain dielectric whose charging could influence device operation. However tests on accelerometers showed a change in calibration at doses of $100 \mathrm{krad}$ (though the devices remained functional) [27,28]. The failures were due to trapped charge in dielectric films. These doses are for unpackaged devices so that the sensor element is directly irradiated. Similar doses on packaged devices would lead to significantly less damage.

RF switches [29] showed no change in operation at does of up to $150 \mathrm{kRad}$ for one design, but for a different design the device's calibration started to slowly change at doses of $10 \mathrm{kRad}$, although the device continued to operate after doses of $300 \mathrm{kRad}$, but with an $80 \%$ increase in required drive voltage. The difference in dose required for degradation between the two devices is due to the different location of the dielectric layers.

The difference in sensitivity to radiation of the devices discussed above is due to the different impact that trapped charge in dielectric will have on different actuations schemes and MEMS devices. Reference [18] contains a detailed discussion of possible geometry changes, charge dissipation layers, and other approaches that can minimize the effect on device performance of charge that is trapped in dielectric films. The source of trapped charge in ref [18] is not from radiation but from a high $\left(10^{8} \mathrm{~V} / \mathrm{m}\right)$ applied electric field, but the reasoning and mitigation techniques are the same.

Figures $2 \mathrm{a}$ and $2 \mathrm{~b}$ (from reference [18]) are schematic cross-section of two micromirror devices. The black dots represent trapped charge. Fig $2 \mathrm{a}$ shows the simpler geometry that is susceptible to performance degradation due to trapped charge. Fig $2 \mathrm{~b}$ shows an improved geometry where all the dielectric not screened by conductive electrodes was selectively etched so that any trapped charges no longer affect the micro-mirror tilt angle. This bottom design can cope with large radiation doses with no change in device characteristics.

Similar strategies for mitigating charge build-up in dielectric layers can be employed to further radiation harden MEMS devices. The AF for radiation is principally total radiation dose. 

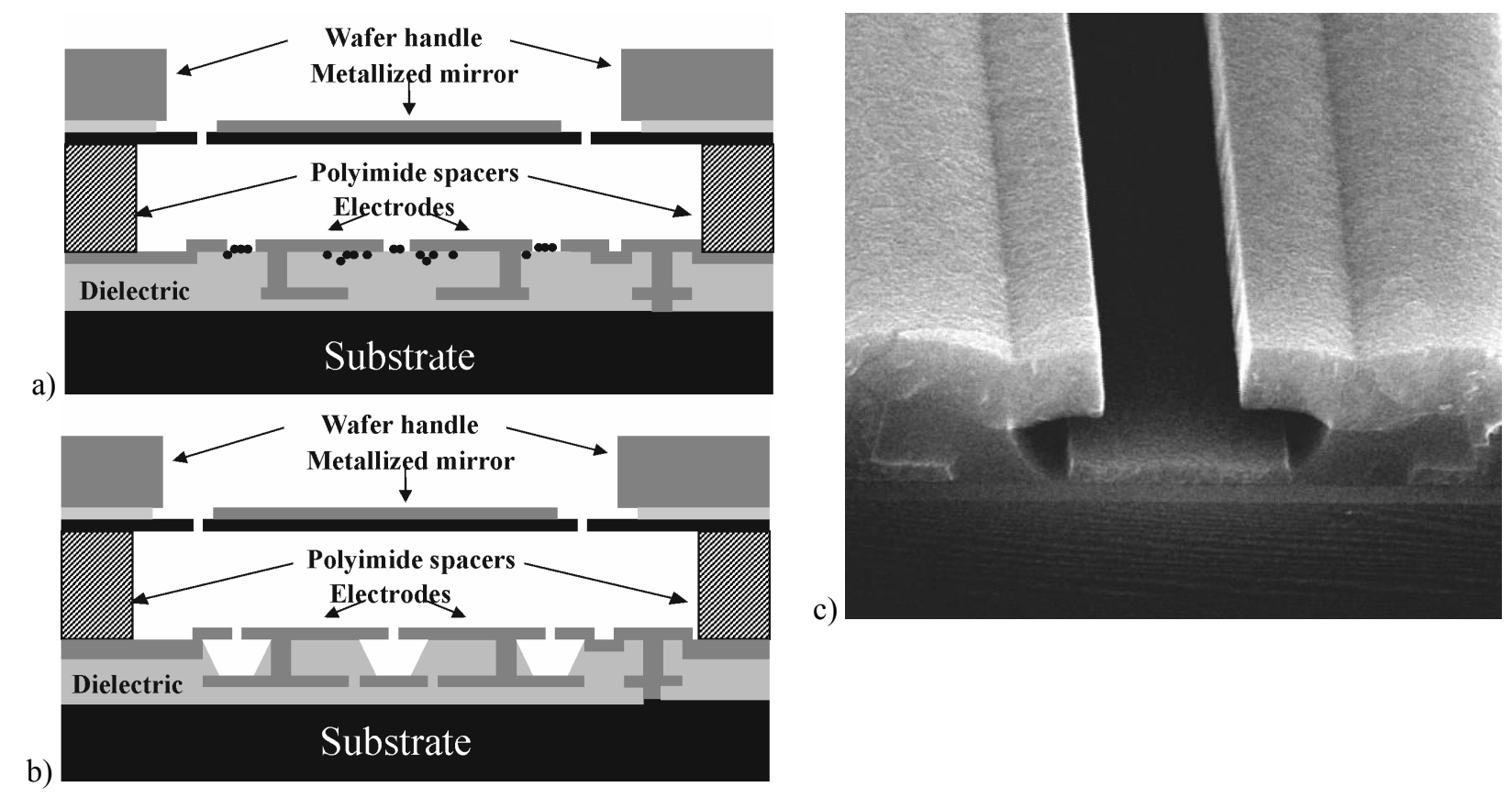

Figure 2 (from reference [18]). a) Schematic cross-section of an electrostatically actuated micromirror that is susceptible to performance degradation due to trapped charge in the dielectric. b) Improved geometry with selective etch to remove all electrically unshielded dielectric so that electrostatic performance is no longer affected by trapped charge. c) SEM micrographs of an electrode chip as in the bottom of Fig 2b, showing the gap between two-level poly-Si electrodes, with the exposed oxide between levels removed by wet etch (buffered HF). The electrodes (structures on the right and left of the micrograph) are spaced by $2 \mu \mathrm{m}$. Rather than leaving oxide between the electrodes, the bottom of the gap between electrodes is covered by a grounded strip of poly-Si (running up the center of the image). The electrical potential of all surfaces is well defined, and the MEMS mirror is shielded from trapped charge in any remaining oxide. Drift in tilt angle due to dielectric charging is completely eliminated.

\subsection{Vacuum}

Devices in space operate in an extremely high vacuum. From a MEMS device perspective, operation in vacuum is only an issue if one of two conditions are met: a) the device is not hermetically packaged, or b) the device generates (or absorbs) more than a few $\mathrm{mW}$ of heat.

Both those conditions are very unlikely. Power dissipation by a MEMS device is usually of order a few microwatts (except for thermally actuated MEMS). Special cases where cooling of the device is required (for example if using a MEMS micromirror to steer a high-power laser beam) can usually be dealt with by packaging the MEMS device hermetically with an exchange gas to allow for convective cooling, and then cooling the package.

For space applications (where the cost of a truly hermetic package is negligible compared to the cost of testing) it is the opinion of the author that all MEMS devices must be hermetically packaged. A true hermetic package with welded or soldered seals (no glues or adhesives because they are permeable to gases including water vapour) can guarantee that the device will operate under a well defined atmosphere for times in excess of 20 years. This is particularly important for damping: a well defined pressure of a gas is often used to achieve critically damped or overdamped behaviour to avoid ringing. Devices designed to operate in vacuum anyway require a hermetic package to allow for testing on earth in laboratory conditions. Having the MEMS device in a hermetic packaging avoids any possible issue of outgassing, mass loss and contamination of other surfaces on the satellite due to the MEMS device, since the MEMS device is not exposed to the vacuum of space.

The Paschen curve describes a simple model for breakdown voltage (arcing) vs. gap and gas pressure between metal plates. This model is overly simplistic for MEMS devices with gaps of order 3 microns or less, for which the modified 
Paschen curve [30] must be used to account for field emission. A controlled gas pressure and chemistry also helps control arcing, providing yet another reason for a hermetic package.

AFs include vacuum tests, coupled with thermal shocks under vacuum.

\subsection{Thermal shocks}

Thermal shock for MEMS, especially those used in picosats in LEO orbits, could be quite severe, of order 16 cycles from $-80 \mathrm{C}$ to $+100 \mathrm{C}$ per day, with possibly much larger temperature excursions possible depending on the orbit and mission (e.g., Mercury orbiter, Mars lander).

Assuming that the materials have been appropriately chosen for the maximum temperature (for example Aluminum up to $200 \mathrm{C}$ with a low strain design, Silicon up to $350 \mathrm{C}$, $\mathrm{SiC}$ up to $800 \mathrm{C}$ ), the biggest challenge is the mismatch in Coefficient of Thermal Expansion (CTE) either between the components of the MEMS device, or between the MEMS device and package (i.e., die attach failure). Thermal shocks can lead to failure of the die bond, cracking of the chip, and to delamination of the layers in the MEMS devices. The curvature of free-standing surfaces consisting of more than one material (such as a metal coated cantilevers) can also change, leading to device failure.

For MEMS level delamination, one solution is to use a monolithic process so all materials have the same CTE. Another is to assemble the device from materials with same CTE; this can be a technologically difficult solution. For device to package level reliability, one should try to make use of compliant attachment schemes and to include stress relief in the design of the microsystem.

Accelerated testing is straightforward: repeated thermal shocks with temperatures exceeding the expected ranges, but by far more than the 10 degrees suggested for qualification in ECSS-E-10-03 [31]. The device should be operating during the thermal shocks, and its characteristics continuously monitored for any sign of degradation.

\subsection{Vibration and Mechanical Shock}

The vibrations experienced during launch and the mechanical shock experienced during separation may be much larger the vibration and shocks experienced in typical industrial applications on earth. The vibration levels depend on the launcher, but let us consider as a representative example a sine vibration from $5 \mathrm{~Hz}$ to $100 \mathrm{~Hz}$ and from 3 to $20 \mathrm{G}$, and shock of $500 \mathrm{G}$ at stage and fairing separation (these values could be much higher for certain missions, for instance up to $10,000 \mathrm{G}$ for $0.1 \mathrm{~ms}$ at stage separation or heat shield ejection using pyrotechnic bolts).

The common perception is that MEMS devices are susceptible to shock and vibration. If care is taken in the design and package, this is not the case. Newton's second law:

$$
\text { force }=\text { mass } * \text { acceleration }
$$

allows us to quickly understand why MEMS can be built to survive very large shocks. Their mass is typically of order micrograms or less, so even shocks of $1000 \mathrm{G}$ produces only $\mathrm{mN}$ forces, and suspension that can handle $\mathrm{mN}$ forces are not that difficult to design for most applications. Commercially available accelerometers from Analog Devices and Colibrys are specified to survive $10^{\prime} 000 \mathrm{G}$ shocks. Sandia National Labs has published data demonstrating MEMS devices that operate after $40,000 \mathrm{G}$ shocks [32]. Of course a poorly designed device will fail sooner, but by creating a symmetrically suspended geometry, avoiding stress concentration (e.g., no sharp corners, only rounded bends), and minimizing strain, one can readily fabricate $\mathrm{mm}$-scale suspended structures that can survive repeated shocks in excess of $1,000 \mathrm{G}$ for $0.5 \mathrm{~ms}$ pulses, see for example ref [33]

Regarding vibration, the most important factor is the coupling of the frequency $\omega$ of the applied vibration with the natural frequency $\omega_{0}$ of the MEMS structure. The applied mechanical force $\mathrm{F}_{0}$ is amplified as:

$$
F=F_{0}\left[\left(1-\frac{\omega^{2}}{\omega_{0}^{2}}\right)^{2}+\left(\frac{1}{Q}\right)^{2} \frac{\omega^{2}}{\omega_{0}^{2}}\right]^{-1 / 2}
$$

where $\mathrm{Q}$ is the quality factor of the particular mechanical mode. 
It is therefore essential to design devices with: either resonance frequencies above the frequencies expected during launch (this is not too challenging since typical resonant frequencies are $500 \mathrm{~Hz}$ to $10 \mathrm{kHz}$ for MEMS, while launch vehicles have their main modes closer to $50 \mathrm{~Hz}$ ), or with $\mathrm{Q}<1$ to eliminate or reduce the force amplification due to the coupling effect. It is advisable to keep the maximum acceleration due to applied vibration well under $100 \mathrm{G}$ to eliminating the possibility of fracture of the MEMS.

\subsection{Packaging}

The importance of a hermetic package for the long-term reliability of MEMS devices has been mentioned several times above. Package failure could lead to a dramatically reduced lifetime. For high reliability applications the packaging costs can often exceed the cost of the device.

The traditional (MIL Spec) definition of a hermetic package is that it contains at most 5,000 ppm of water vapor at the time of initial sealing and that the leak rate is less than $10^{-8}$ atm. $\mathrm{cm}^{3} / \mathrm{sec}$ to prevent entry of significant moisture during the device's expected lifetime. Such a leak rate is a meaningful upper allowable limit for a large package, but can be unacceptably high for the small volumes enclosed in typical MEMS packages, for which a different definition and more careful leak testing must be performed.

Hermetic packages are made from ceramics and metals, with possibly glass or sapphire for optical access. Since all materials are permeable to a certain extent to gasses, true hermeticity can never be achieved. However since the diffusion of water vapor through metals and silicon is several orders of magnitude smaller than diffusion through polymers, by suitable choice of packaging materials one can maintain a well controlled environment over a 20 year product life. There may be no glued seals: all seals are welded or soldered. Getters can be used if necessary, but they typically require thermal activation, and can produce particulates.

Thermal shock testing of the package itself is important to accelerate any leakage due to cracked or failed seams. The package being tested need not include an active device. Ideally a humidity sensor is included in the test packages. One alternates between a series of thermal shocks (for example $-85 \mathrm{C}$ to $+120 \mathrm{C}$ to accelerate package failure) with exposing the package to $85 \%$ humidity at $85 \mathrm{C}$ (to allow moisture to enter). One then looks for moisture ingress by measuring the humidity level in the package. A more sensitive (but destructive) technique is to perform the accelerated tests on the package, and then do a residual gas analysis on the package contents: this has the advantage of identifying all substances may have outgased from the interior of the package, as well as possible moisture or air ingress.

An alternative to a standard hermetic package is to perform the packaging on the chip- or wafer- level, using wafer bonding techniques (such as fusion or anodic bonding): a cap wafer or chip is hermetically bonded onto the MEMS device chip or wafer. This cap can be a Silicon wafer (for example for accelerometers) or a glass wafer (for example for optical MEMS). Wafer-scale packaging offers many advantages including lower cost (no expensive ceramic packages and is a batch process), and requires much less space and weighs much less than a conventional package.

Despite these advantages, wafer-level packaging is still in its infancy for MEMS, as it requires a more complex fabrication scheme than traditional packages and imposes a stricter thermal budget during fabrication.

\section{CONCLUSION}

The growing acceptance of MEMS in safety critical application on earth (most notably accelerometers for airbags systems, and also IMUs) as well as in consumer electronics (projection displays) is a testament to the high level of reliability that can be achieved in suitably designed and packaged MEMS.

Space presents a unique environment that may lead to additional failure modes, for instance due to radiation. A fundamental physics-based understanding of the failure modes is required to be confident that the accelerated tests provide an accurate prediction of device lifetime. While details depend on the specifics of each MEMS design, MEMS is a sufficiently mature field that all major failure modes are now well understood. The techniques for accelerated testing of MEMS are well established (except for stiction and shelf-life). Qualifying COTS MEMS for space can follow a very similar approach to qualifying COTS microelectronic devices for space.

The package plays a key role in ensuring the long-term reliability of a MEMS device. For space applications (where the cost of a truly hermetic package is negligible compared to the cost of testing) all MEMS devices must be hermetically packaged. 


\section{Acknowledgements}

Portions of this work were performed while HS was with Bell Labs, Lucent Technologies. HS wishes to thank in particular Arman Gasparyan, Susanne Arney, and Ho Bun Chan.

\section{REFERENCES}

1. For example Analog Devices accelerometer model ADXL78: http://www.analog.com/ UploadedFiles/Data_Sheets/50606390ADXL78_a.pdf

2. For example Freescale pressure sensor model MPXM2010

3. Texas Instrument's DMD chip. See http://www.dlp.com/

4. "MEMS for space applications: a reliability study", S. Barthe, F. Pressecq, L. Marchand, 4th Round Table on MNT for Space. 20-22 May 2003, ESTEC, Noordwijk, Netherlands. Available at: https://escies.org/public/mnt4/

5. "Reliability of COTS MEMS Accelerometer Under Shock And Thermomechanical Cycling ", by Reza Ghaffarian, 2001 SMTA International Conference

6. "Evaluation of Thermo-Mechanical Stability of COTS Dual-Axis MEMS Accelerometers for Space Applications", by A. K. Sharma and A. Teverovsky, Proceedings EEE Links Electronic Packaging and Space Parts New January 2001

7. ECSS-Q-60-11A: Derating and end-of-life parameter drifts - EEE components, (European Cooperation for Space Standardization) available at: http://www.ecss.nl/

8. "Overview of MEMS/NEMS technology development for space applications at NASA/JPL", by T. George, Proceedings of SPIE, Proc. SPIE Int. Soc. Opt. Eng. 5116, 136 (2003)

9. www.teravicta.com

10. "Microelectromechanical system radio frequency switches in a picosatellite mission", by J.Yao et al., Smart Mater. Struct. 10, p.1196-1203, 2001

11. "Reliability of RF-MEMS", I. De Wolf et al., 4th Round Table on MNT for Space. 20-22 May 2003, ESTEC, Noordwijk, Netherlands. Available at: https://escies.org/public/mnt4/

12. "Reliability Assessment and Lifetime Testing with Micro-Mirrors", S. Manhart et al., 4th Round Table on MNT for Space. 20-22 May 2003, ESTEC, Noordwijk, Netherlands. Available at: https://escies.org/public/mnt4/

13. "Micropropulsion for small spacecraft: a new challenge for field effect electric propulsion and microstructured liquid metal ion sources", by J. Mitterauer. Surface and Interface Analysis, Vol. 36, Issue 5-6 , p. 380 - 386 (2002)

14. "Digital MicroPropulsion", Lewis, et al. Sensors \& Actuators A, 2000, 80(2), p.143-154

15. "A hybrid cold gas microthruster system for spacecraft", J. Köhler, J. Bejhed, H. Kratz, F. Bruhn, U. Lindberg, K. Hjort, L. Stenmark, Sensors and Actuators A (Physical), A97-98:587-98, April 2002.

16. "Integrated System To Analyze The Genetic Effects Of The Space Environment On Living Cells In Culture: Genesat", by A. J. Ricco1 et al., p. 527, Proceedings of the $9^{\text {th }}$ International conference on miniaturized systems for Chemistry and Life Science ( $\mu$ TAS 2005), Boston, October 2005

17. "Designing for MEMS Reliability", by S. Arney, MRS Bulletin, April 2001, p. 296

18. "Effects of Electrical Leakage Currents on MEMS Reliability and Performance", by H. Shea et al., IEEE Transactions on Device and Materials Reliability, Vol. 4, No. 2, p.198, 2004

19. "MEMS reliability, characterization, and test", by A. Hartzell et al., Proc. SPIE Vol. 4558, p. 1-5, Reliability, Testing, and Characterization of MEMS/MOEMS, R. Ramesham; Ed. 2001.

20. "Designing MEMS for Reliability", short course by S. Arney, A. Gasparyan, and H. Shea, given at SPIE Photonics West, Jan 2001

21. "Mechanism of fatigue in micron-scale films of polycrystalline silicon for microelectromechanical systems", C. Muhlstein et al., Appl. Phys. Lett., Vol. 80, No. 9, 4 March 2002 p.1532

22. "High-Cycle Fatigue of Single-Crystal Silicon Thin Films", C. Muhlstein et al., Journal Of Microelectromechanical Systems, Vol. 10, No. 4, December 2001, p. 593

23. "Beam-Steering Micromirrors for Large Optical Cross-Connects", by V. A. Aksyuk, Journal Of Lightwave Technology, Vol. 21, No. 3, March 2003, p. 634

24. "Tensile testing of MEMS materials—recent progress", W. N. Sharpe, Jr., J. Bagdahn, K. Jackson, G. Coles, Journal of Materials Science 38 (2003) p.4075

25. "Modeling of fatigue in polysilicon MEMS structures", by K. Bhalerao, A.B.O. Soboyejo, and W.O. Soboyejo, Journal Of Materials Science 38 (2003) p.4157 - 4161 
26. "Radiation effects on surface micromachined comb drives and microengines," L. P. Schanwald et al., IEEE Trans. Nucl. Sci., vol. 45, no. 6, pp. 2789-2798, 1998.

27. "Radiation Response of a MEMS Accelerometer: an Electrostatic Force", by L. Edmonds et al, IEEE Transactions on Nuclear Science, Vol 45, No. 6, p. 2779, 1998

28. "Total Dose Effects on Microelectromechanical Systems (MEMS): Accelerometers", by C. I. Lee et al., IEEE Trans. Nucl. Sci, Vol. 43, No. 6, Dec. 1996, p.3127

29. "Radiation Effects in Micro-Electromechanical Systems (MEMS): RF Relays", by S. McClure et al., IEEE Trans. Nucl. Sci., Vol. 49, No. 6, Dec. 2002 p. 3197

30. Electrical breakdown and ESD phenomena for devices with nanometer-to-micron gaps, " A. Wallash and L. Levit, in Reliability, Testing, and Characterization of MEMS/MOEMS II , R. Ramesham and D. M. Tanner, Eds., 2003, vol. 4980, Proc. SPIE, pp. $87-96$

31. ECSS-E-10-03 : Testing, (European Cooperation for Space Standardization) available at: http://www.ecss.nl/

32. "MEMS Reliability in Shock Environments", by D. Tanner et al., Proceedings of 38th annual Reliability Physics Symposium (IRPS), 2000, p. 129

33. "Drift-Free, 1000G Mechanical Shock Tolerant Single-Crystal Silicon Two-Axis MEMS Tilting Mirrors in a 1000x1000-Port Optical Crossconnect", by A. Gasparyan, et al, Post deadline paper PD36-1, Optical Fiber Communication Conference and Exhibit 2003, OFC 2003, March 2003 OFC Atlanta, GA 